\title{
A REAÇÃO DE DIELS-ALDER NO INÍCIO DO SÉCULO VINTE UM
}

Timothy John Brocksom,* Maria Carolina Donatoni, Marciana Pierina Uliana e Ygor William Vieira

Departamento de Química, Universidade Federal de São Carlos, CP 676, 13560-970 São Carlos - SP, Brasil

Recebido em 29/6/10; aceito em 23/8/10; publicado na web em 20/10/10

THE DIELS-ALDER REACTION AT THE BEGINNING OF THE TWENTY-FIRST CENTURY. The Diels-Alder reaction continues to be the premier method for the construction of complex organic molecules. In the last 10 years many developments have been introduced, and have led to increased utility of this reaction. In this review we present some of these novelties, which are of fundamental importance in organic synthesis.

Keywords: Diels-Alder; Diels-Alderase; multi-component reactions.

\section{INTRODUÇÃO}

Desde a nossa revisão em $2001,{ }^{1}$ houve a publicação de pelo menos 11.500 artigos tratando especificamente da reação de DielsAlder. ${ }^{2}$ Numa média de aproximadamente 1.200 publicações por ano, tentaremos focalizar nossa atenção nas inovações realmente relevantes, destacando que estamos tratando de uma reação clássica que, porém, sempre se renova. As inovações se encontram em aspectos teóricos, metodologias novas e aplicações em síntese total.

Em 2002, a revista Angew. Chem., Int. Ed. (vol. 41, n. 10) destacou na sua capa esta reação com fotografias dos dois químicos alemães, lembrando o centenário do nascimento de Alder. Foram publicadas revisões por Corey sobre catálise enantiosseletiva envolvendo ácidos de Lewis, ${ }^{3}$ e o grupo de Nicolaou apresentou as suas aplicações em síntese total. ${ }^{4}$

No mesmo ano Fringuelli e Taticchi lançaram uma monografia tratando dos aspectos mais convencionais da reação até aquele momento. ${ }^{5}$ Orbitais de fronteira ganharam destaque com dois livros, incluindo reações pericíclicas, e obviamente cicloadições [4+2], em um nível acessível aos químicos orgânicos sintéticos. ${ }^{6,7}$

No Esquema 1 encontra-se a reação clássica entre um dieno conjugado $^{8}$ (ciclopentadieno) e um dienófilo com grupo retirador de densidade eletrônica (EWG) (para-benzoquinona), como ocorre na grande maioria das reações encontradas na literatura. $\mathrm{O}$ produto cicloaduto é um cicloexeno altamente funcionalizado, com até quatro centros estereogênicos novos, sendo criadas simultaneamente duas ligações $\sigma$ novas. É digno de nota que as reações pericíclicas em geral, e certamente as cicloadições [4+2], as reações de Diels-Alder, não precisam de grupos protetores para a sua perfeita execução (conceito de Baran), ${ }^{9}$ ocorrem com total economia de átomos (conceito de Trost),${ }^{10}$ apresentam um aumento considerável de complexidade

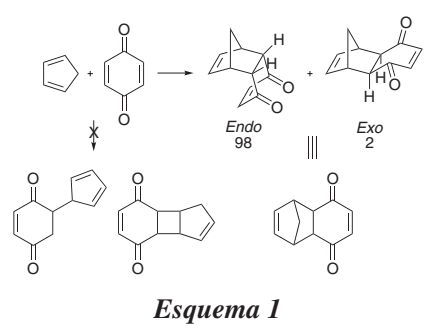

*e-mail: brocksom@ @erra.com.br

\#Artigo dedicado ao Prof. Dr. Hans Viertler, nosso grande amigo, colega, professor e conselheiro, por ocasião de seus 70 anos. estrutural (conceito de Wender) ${ }^{11}$ e com a criação de duas ligações $\sigma$ novas são altamente construtivas (conceito de Hendrickson). ${ }^{12}$

Atualmente, a reação de Diels-Alder compete com as reações de metátese ${ }^{13,14}$ e acoplamento cruzado ${ }^{15}$ como as reações chaves em síntese total, frequentemente em parceria ou até em combinação dominó. ${ }^{16,17}$

Outro aspecto novo se refere à abordagem dada à reação de DielsAlder em livros textos. Geralmente a reação era tratada como uma reação muito relevante, mas, dentro da química de alcenos e dienos conjugados, enquanto agora, criam-se capítulos especiais sobre reações pericíclicas com o destaque da cicloadição [4+2]. É interessante constatar como esta nova abordagem se apresenta hoje em alguns livros textos de graduação ${ }^{18} \mathrm{e}$ de pós-graduação. ${ }^{19}$ Uma prova desta afirmação pode ser encontrada em um experimento computacional, proposto para alunos de graduação, da reatividade relativa de reações de Diels-Alder de diversas 2-ciclo-exenonas com ciclopentadieno, desenvolvido por Lacerda Jr. e colaboradores.$^{20}$ Sem dúvida, a disponibilidade de programas computacionais comerciais como PCModel, Hyperchem e Gaussian $2003^{\circledR}$ contribui para difundir esta prática.

Recentemente surgiu a informação de que Corey reclama para si a autoria intelectual da teoria da simetria de orbitais em reações eletrocíclicas, ${ }^{21}$ e que passou esta sugestão para Woodward, dentro do seu convívio em Harvard. Como Woodward faleceu em 1979, a polêmica ficará aberta, apesar de que Hoffmann nega esta versão baseado em conversas com Woodward. ${ }^{22}$

\section{O MECANISMO E ASPECTOS TEÓRICOS}

A reação de Diels-Alder é alvo constante de estudos buscando um entendimento melhor de seu mecanismo, aliando cálculos computacionais aos resultados experimentais. A reação de Diels-Alder é dita uma reação pericíclica de cicloadição [4+2] entre um dieno conjugado com quatro elétrons $\pi$ e um segundo componente alceno com dois elétrons $\pi$, chamado de dienófilo, em uma reação concertada, mas provavelmente assincrônica, e em uma relação suprafacial-suprafacial. Esta frase complicada quer dizer basicamente que a cicloadição se procede numa única etapa, sem intermediários, por um único estado de transição, mas neste estado de transição pode haver a formação das duas ligações químicas covalentes com uma diferença temporal entre as duas. Por extensão, incluem também reações de cicloadição de Diels-Alder em que o dieno e o dienófilo são fortemente polarizados e em que o mecanismo pode ser iônico e não pericíclico (Figura 1). ${ }^{23}$

Dentro das regras de Woodward-Hoffmann, ${ }^{24}$ da teoria de Fukui de orbitais de fronteira (FMO) ${ }^{25}$ e os coeficientes dos orbitais atômi- 


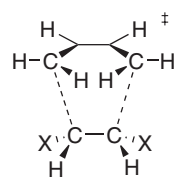

Sincrônico
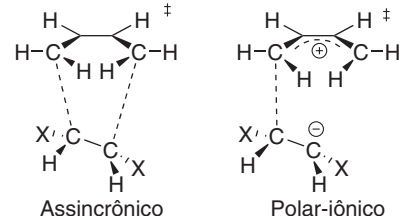

Figura 1 cos dos dois componentes dieno e dienófilo, ${ }^{7}$ é possível prever com bastante sucesso as estruturas dos produtos principais de qualquer cicloadição quanto às questões de quimiosseletividade, regiosseletividade e estereosseletividade (diastereo- e enantio-). O conceito de interações secundárias de orbitais, utilizado para explicar a preferência cinética do estado de transição endo sobre o exo, foi questionado e logo a seguir defendido. ${ }^{26,27}$

Um exemplo importante da relevância de cálculos aparece no caso da primeira reação estudada, entre ciclopentadieno e parabenzoquinona (Esquema 1). Inicialmente, Albrecht e outros atribuíram estruturas de adição nucleofílica 1,4 e cicloadição [2+2] e, finalmente, Diels e Alder propuseram a estrutura correta sem definir a estereoquímica relativa. Um estudo mais moderno por Yates e Switlak ${ }^{28}$ definiu experimentalmente os dois cicloadutos obtidos, as suas estereoquímicas relativas e a interconversão do endo para o exo. Entretanto, esta interconversão é muito pouco desenvolvida (aproximadamente 98:2), apesar das afirmações contundentes em livros textos para o caso clássico de ciclopentadieno e o anidrido maleico. Em um estudo teórico recente, ${ }^{29}$ Tormena, Lacerda Jr. e de Oliveira demonstraram a maior estabilidade relativa do produto endo neste caso e, assim, este produto é simultaneamente o cinético e o termodinâmico. Finalmente, os resultados experimentais de Yates e Switlak encontram embasamento teórico. Agora precisamos checar outros casos, inclusive de ciclopentadieno e anidrido maleico!

A questão do mecanismo da reação entre dienos com grupos retiradores de densidade eletrônica (EWG) e dienófilos com grupos doadores de densidade eletrônica (EDG), denominada de demanda inversa eletrônica, foi investigada pelo grupo de Spino com conclusões surpreendentes. ${ }^{30}$ Os autores conseguiram demonstrar que as duas interações de fronteira na Diels-Alder não são equivalentes, que a diferença $\mathrm{HOMO}_{\text {dienófilo }}-\mathrm{LUMO}_{\text {dieno }}$ deve ser utilizada com cuidado para prever a reatividade e que a forma assincrônica do estado de transição deve ser levada em consideração. É demonstrado também que os dienófilos polarizados são mais reativos do que os não polarizados, e confirmam que os coeficientes orbitalares podem ser usados para compreender a reatividade relativa e não apenas a regioquímica da cicloadição.

Ogawa e Fujimoto investigaram novamente a interação dos orbitais na autocicloadição do butadieno ${ }^{31}$ e demonstraram que o efeito das interações orbitalares secundárias deve ser muito menos significante do que se tem assumido (Figura 2). A confirmação foi obtida por análises numéricas a respeito do estado de transição endo da reação entre butadieno e anidrido maleico, onde as interações orbitalares secundárias aparecem quando vários orbitais moleculares ocupados e desocupados são levados em consideração.
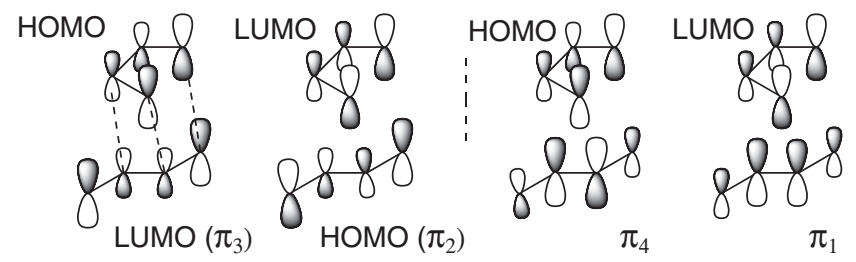

Figura 2
O grupo de Houk desenvolve uma pesquisa onde os cálculos são relacionados com experimentos feitos anteriormente, ou junto com outro grupo de pesquisa experimental. Exemplos recentes incluem a relação do produto do estado de transição endo versus exo na reação de dienos sem e com um grupo metílico no carbono terminal (Esquema 2), em colaboração com o grupo de Gouverneur; ${ }^{32}$ a enantiosseletividade de cicloadições muito simples utilizando o catalisador de oxazaborolidina de Corey (Esquema 3) ${ }^{33}$ e a regiosseletividade da reação entre o vinil-indeno e mono cetais de para-benzoquinonas com o grupo de Danishefsky (Esquema 4)..$^{34}$

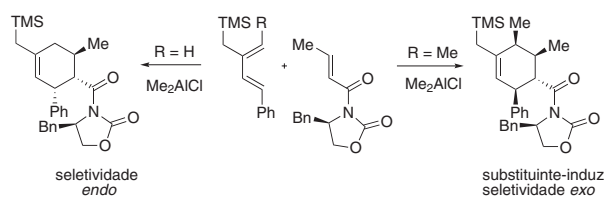

Esquema 2

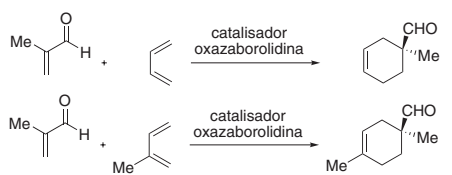

Esquema 3

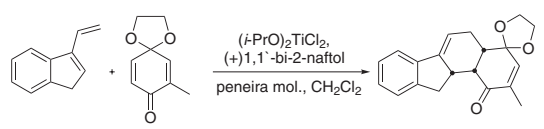

Esquema 4

\section{REAÇÕES DE DIELS-ALDER NA NATUREZA: DIELS- ALDERASE}

A natureza também já descobriu as vantagens de incorporar as reações pericíclicas para a construção de moléculas orgânicas complexas naturais, conhecidas como metabólitos secundários. Em um caso antológico, dos ácidos endiandricos, ${ }^{16}$ há uma sequência de reações pericíclicas incluindo duas reações eletrocíclicas e uma Diels-Alder intramolecular. Estes produtos naturais possuem oito centros estereogênicos, mas ocorrem na natureza como racematos e, assim, sugere-se que são formados por meios não enzimáticos.

Assim a discussão continua sobre diversos produtos naturais evidentemente formados por reações pericíclicas, mas realmente envolvem enzimas ou são espontaneamente gerados? Em 2003, Stocking e Williams apresentaram uma revisão sobre a reação de DielsAlder na biossíntese das principais classes de produtos naturais ${ }^{35}$ e, em 2004, Oikawa e Tokiwano publicaram uma revisão com 280 referências sobre o mesmo assunto. ${ }^{36}$ Alguns exemplos são apresentados na Figura 3, incluindo lovastatina, macrofomato, solanapirona A, chaetoglobosina A, spinosina A e clorotricina. ${ }^{37}$

Watanabe descreve as possibilidades de biotecnologia com os conhecimentos adquiridos na biossíntese de produtos naturais pela reação de Diels-Alder. ${ }^{38}$ Kelly questiona o número muito baixo de Diels-Alderases realmente identificados, dentro da biossíntese de policetídeos por reações intramoleculares. ${ }^{37}$

Em 2005, Gouverneur relata um exemplo de um anticorpo que catalisa uma reação de hetero-Diels-Alder que conduz o aduto trans, dentro da procura de hetero-Diels-Alderases por biocatálise (Esquema 5). ${ }^{39}$

A elucidação da primeira estrutura cristalina de uma enzima Diels-Alderase gerou dois caminhos possíveis e diferentes para o áci- 


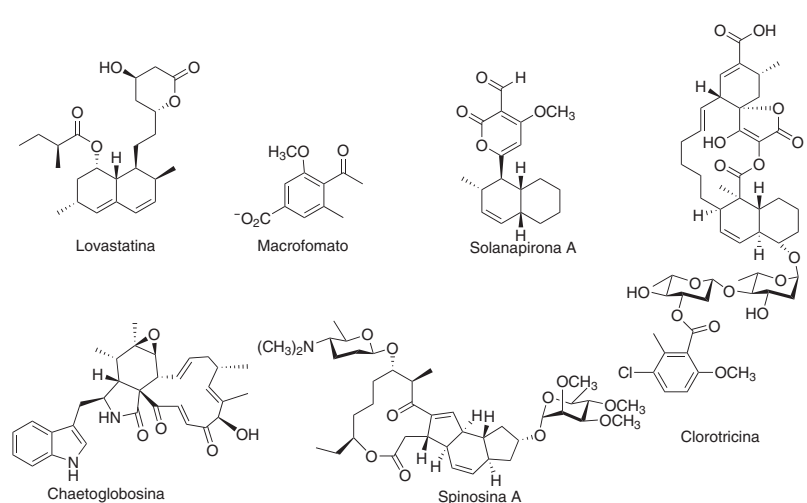

Figura 3

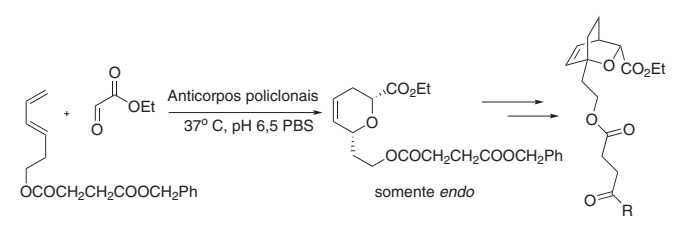

Esquema 5

do macrofomico, onde uma rota envolve uma sequência de Michaelaldol, e a outra uma reação de Diels-Alder (Esquema 6).$^{40} \mathrm{O}$ grupo de Jorgensen calculou as energias de ativação por estes dois caminhos e concluiu que o processo Michael-aldol é o de menor energia. ${ }^{41}$

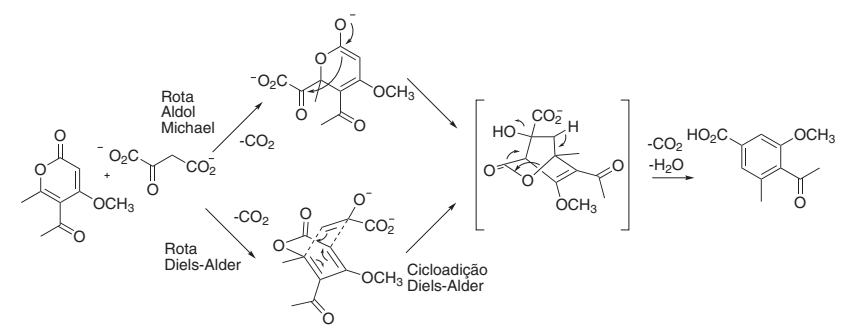

Esquema 6

\section{METODOLOGIAS}

\section{Catálise com ácidos de Lewis}

Nunes e Bieber utilizaram pentacloreto de antimônio para catalisar as reações entre simples dienos e para-benzoquinonas, demonstrando uma interessante inversão de regioquímica frente à reação conduzida em condições térmicas normais (Esquema 7). ${ }^{42}$

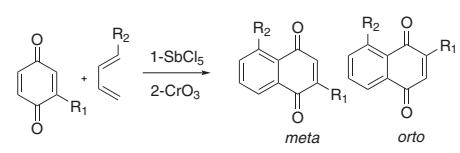

Esquema 7

O grupo de Constantino vem utilizando o pentacloreto de nióbio, a baixa temperatura, para melhorar e modificar seletividades nas cicloadições de cicloalquenonas conjugadas. Somente as enonas substituídas no carbono $\beta$ não reagem, enquanto as demais demonstram excelentes resultados (Esquema 8). ${ }^{43,44}$

Uma aplicação interessante de ligação de hidrogênio para catalisar uma reação enantiosseletiva de hetero-Diels-Alder entre dienos ativados e aldeídos está mostrada no Esquema $9 .{ }^{45} \mathrm{O}$ catalisador é simplesmente um álcool TADDOL quiral e enantiopuro, e o cicloa-

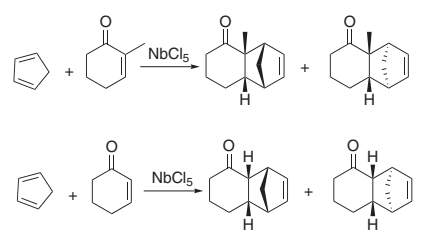

Esquema 8

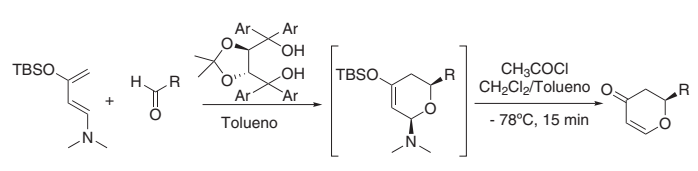

Esquema 9

duto é hidrolisado a di-hidropirona em pelo menos $98 \%$ de excesso enantiomérico.

\section{Organocatálise enantiosseletiva}

O grupo de MacMillan vem contribuindo de forma fundamental para o desenvolvimento desta nova (ou redescoberta) área de catálise de reações orgânicas em geral e, especificamente, as reações de Diels-Alder. ${ }^{46}$ Merino e colaboradores publicaram em 2010 uma revisão sobre organocatálise enantiosseletiva em reações de DielsAlder, o que dispensa maiores comentários aqui. ${ }^{47}$ Esta revisão faz uma abordagem dos diversos tipos de moléculas orgânicas que são utilizadas como catalisadores, tais como aminas quirais, imidazolidinonas, aminoácidos como a L-prolina, hidrazinas cíclicas, sulfonil hidrazinas, binaftil diaminas, alcaloides Chincona, carbenos heterocíclicos, e guanidinas.

Uma modificação relevante é o uso de organocatalisadores suportados em polímeros, ${ }^{48}$ como a L-prolina, e as imidazolidinonas de MacMillan, como mostrado no Esquema 10. Outro exemplo é a reação do cicloexadieno com o acrilaldeído, estudada na presença do organocatalisador suportado em montmorillonita (Esquema 11). ${ }^{49}$

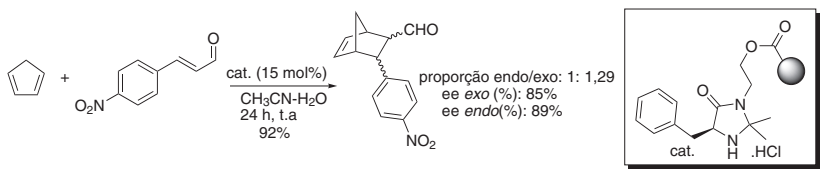

Esquema 10

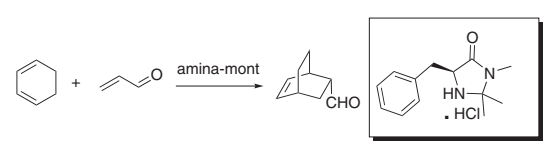

Esquema 11

\section{Micro-ondas}

O uso de micro-ondas em reações de Diels-Alder vem crescendo tremendamente na última década, e agora há evidências que microondas atuam por puro aquecimento e sem os efeitos do espectro eletromagnético da região. ${ }^{50,51}$ Resta então o aquecimento muito mais rápido na região inteira do recipiente e de forma muito mais eficiente. Um aspecto importante na utilização de micro-ondas é a limitação de escala, o que está sendo superado agora. ${ }^{52,53}$ Nesta última citação, Kappe apresenta referências bem recentes, incluindo vários livros e revisões sobre este assunto de crescente importância em química orgânica sintética. A seguir, apresentamos alguns exemplos da utilização de micro-ondas em síntese. 
A síntese total do alcaloide scorpinona, tem como etapa chave uma reação de Diels-Alder regiosseletiva assistida por micro-ondas (Esquema 12). ${ }^{54}$

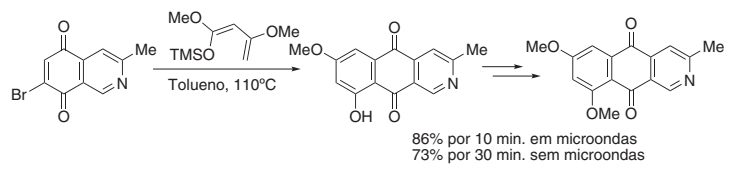

Esquema 12

Um estudo comparativo da reação de Diels-Alder enantiosseletiva em solução e em suporte sólido foi realizado usando 1-aminodienos e acrilatos (Esquema 13). ${ }^{55}$

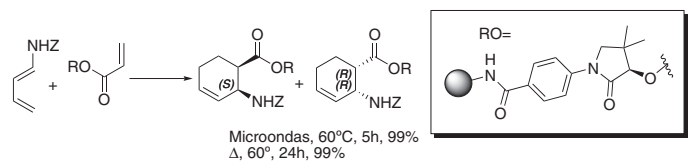

Esquema 13

O grupo de Leadbeater estudou o uso de micro-ondas para se realizar reações orgânicas em escalas maiores, em um reator do tipo multímodo em frascos de teflon selados com capacidade para 350 $\mathrm{mL}$ com temperatura monitorada por sensores de fibra óptica. ${ }^{52} \mathrm{~A}$ reação de Diels-Alder entre isopreno e anidrido maleico foi testada numa escala de 0,1 mol (Esquema 14).

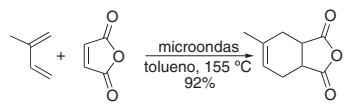

Esquema 14

Moreno e colaboradores mostraram que a reação de DielsAlder de $N$-tosyl-3-nitro-indol com diferentes dienos substituídos ou não com grupos doadores de elétrons, sob efeito de micro-ondas e na ausência de solventes (Esquema 15), fornece derivados de carbazol cuja estrutura está relacionada a alguns produtos naturais, tais como a (-)-aspidospermina e alcaloides plumeranos. ${ }^{56}$

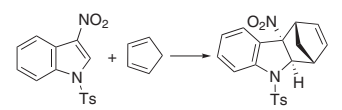

Esquema 15

Antecipando a questão de reações multicomponentes (MCR), Botta e colaboradores reportaram a formação de 2,3-di-hidropiranos, ${ }^{57}$ feita na presença de um catalisador de Grubbs entre um alcino e um alceno, levando a um dieno que reage com um aldeído, conforme o Esquema 16.

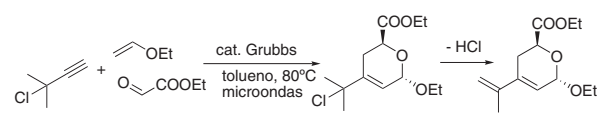

\section{Esquema 16}

Nosso laboratório demonstrou um aumento de rendimento, chegando próximo ao quantitativo, em muito menos tempo, nas cicloadições entre para-benzoquinonas e dienos simples (Esquema 17).$^{58} \mathrm{~A}$ maioria destas reações é conduzida na ausência total de solventes orgânicos.

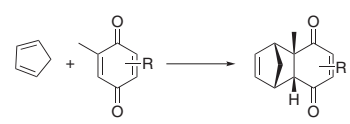

Esquema 17

\section{Reações multicomponentes (MCR)}

As vantagens imensas das reações do tipo multicomponente são evidentes em ganhos de tempo, mas principalmente na eliminação dos sucessivos isolamentos e purificações dos produtos intermediários. Por outro lado, criou-se certa controvérsia com os termos dominó (veja a seguir), em cascata, tandem e zipper. Nosso entendimento é simples, é que a distinção reside no fato que reações multicomponentes exigem pelo menos três substratos presentes desde o início da reação, e partes ou a totalidade destes substratos devem aparecer na estrutura do produto final. Os demais termos se referem às reações que acontecem em sequência, não importando se envolvem um único substrato ou dois.

Ramón e Yus discutem a versão assimétrica desta metodologia (AMCR), ainda chamando de fronteira nova. ${ }^{59}$

Recentemente demonstramos a aplicação da cicloadição entre a timoquinona e um dieno gerado in situ, formando um cicloaduto com o mesmo esqueleto carbônico dos sesquiterpenos eudesmanos (Esquema 18). ${ }^{60}$

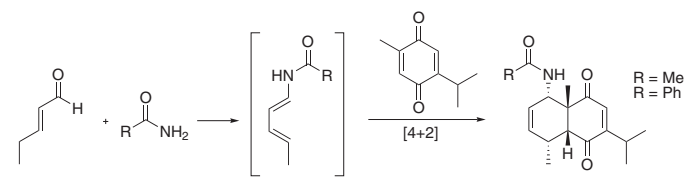

Esquema 18

\section{Reações de Diels-Alder em dominó}

As reações de Diels-Alder em sequência com outras reações de construção de ligações carbono-carbono são denominadas em dominó, apesar de que alguns autores ainda insistem em chamar de tandem, ou em cascata e, eventualmente, zipper. Esta questão já foi abordada por Tietze com muita propriedade, e acreditamos que a nomenclatura já foi resolvida. ${ }^{61}$ Outro aspecto geral envolve a combinação de reações de Diels-Alder em versão multicomponente, utilizando micro-ondas e, ainda, em versão dominó. A literatura sobre estas combinações é muito extensa e só pode ser resumida aqui. Em um número especial da revista Chem. Soc. Rev., 2009, o grupo de Barriault apresentou uma revisão sobre reações pericíclicas em dominó na síntese de compostos carbocíclicos. ${ }^{62}$ Neste mesmo número especial, o grupo de Nicolaou traz de novo a sua visão sobre as reações em cascata. ${ }^{63}$

Em princípio, as reações dominó envolvem a formação do dieno ou a liberação do dienófilo durante uma sequência, e normalmente seria a última reação já que o cicloexeno formado não é propício a efetuar mais reações químicas.

\section{APLICAÇÕES EM SÍNTESE TOTAL}

A discussão aqui será dividida em três partes, iniciando com as reações intermoleculares, para depois apresentar as reações intramoleculares (IMDA) e transanulares (TADA), na síntese total de moléculas orgânicas complexas. Esta discussão não pode esquecer a contribuição pioneira de Desimoni e colegas sobre a síntese total de produtos naturais por meio de reações pericíclicas. ${ }^{64}$

\section{Reações intermoleculares}

O grupo de Constantino vem utilizando o pentacloreto de nióbio 
como catalisador em reações de Diels-Alder com bastante sucesso, e na síntese total da baquenolida-A esta reação ocorre em excelente rendimento, à baixa temperatura e com elevada regiosseletividade (Esquema 19). ${ }^{65}$

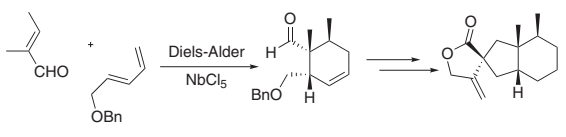

Esquema 19

O grupo de Ferreira desenvolveu uma metodologia de condensação aldólica de lawsona com aldeídos, que depois reage com estirenos numa hetero-Diels-Alder MCR em ótimos rendimentos (Esquema 20). ${ }^{66}$

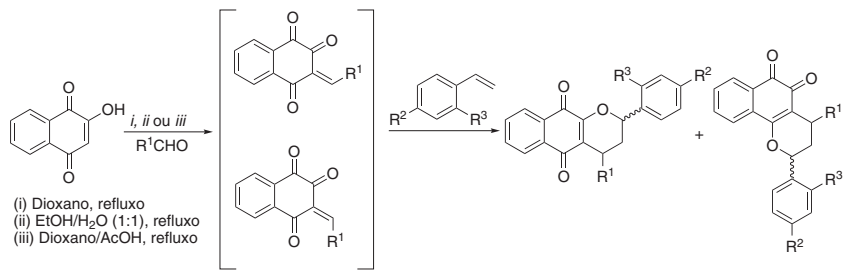

Esquema 20

O nosso grupo desenvolve estudos sobre as reações de Diels-Alder intermolecular entre para-benzoquinonas e dienos simples, tanto em condições clássicas e com o uso de micro-ondas, bem como na versão multicomponente. Este estudo permite sintetizar cicloadutos que são intermediários importantes para sesquiterpenos e diterpenos naturais com elevada atividade biológica (Esquema 21). ${ }^{67}$

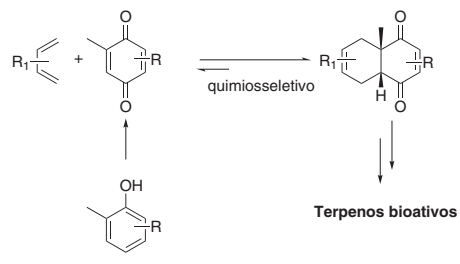

Esquema 21

A reação de Diels-Alder de di-hidro-piridinonas-2 foi efetuada com o dieno de Danishefsky, com rendimento bastante razoável (Esquema 22). ${ }^{68}$

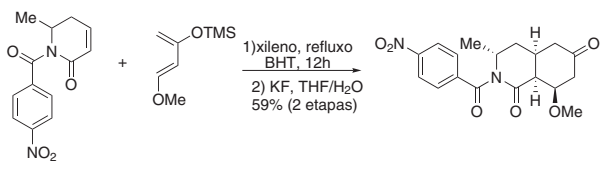

Esquema 22

Liao e colaboradores reportaram a síntese total de alguns eremofilanos (Esquema 23), incluindo a reação de Diels-Alder intermolecular entre uma $o$-benzoquinona mascarada e a etil vinil cetona, levando à construção de um sistema biciclo [2.2.2] octenona e, depois, um rearranjo de Cope produziu um intermediário comum para esta classe de sesquiterpenos. ${ }^{69}$

Em 2007, Danishefsky e colaboradores divulgaram a síntese total da Paecilomicina $\mathrm{A},{ }^{70}$ a partir de uma reação de Diels-Alder intermolecular (Esquema 24), com uma estereosseletividade endo/exo de 10:1.

Matsuda e colaboradores relataram a síntese do sesquiterpeno 10-isociano-4-cadineno isolado de moluscos marinhos da família

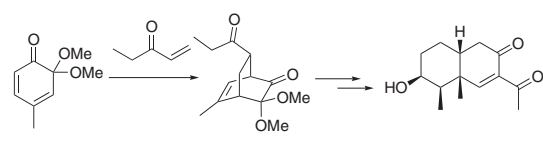

Esquema 23

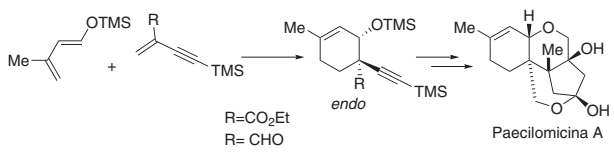

Esquema 24

Phyllidiidae..$^{71}$ A reação de Diels-Alder intermolecular entre o acrilato de metila e um dieno (Esquema 25), na presença de um ácido de Lewis, permitiu a construção de um cicloaduto apropriado para sintetizar o alvo final.

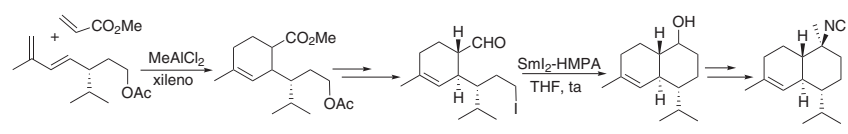

Esquema 25

A molécula de Crisamicina A pode ser analisada sinteticamente como sendo feita por duas unidades contendo um grupo triflato, que seriam obtidas através de uma reação de Diels-Alder (Esquema 26). ${ }^{72}$

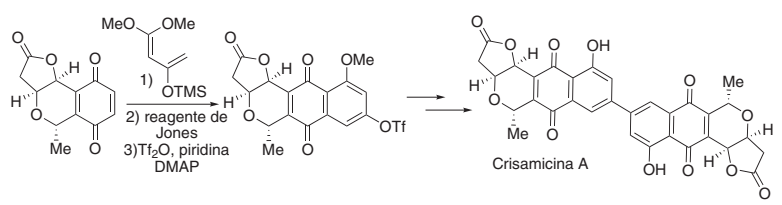

Esquema 26

A síntese do alcaloide 8-oxoerimelantina foi reportada por Yamamoto e colaboradores. ${ }^{73}$ Uma reação de Diels-Alder intermolecular estereosseletiva foi utilizada na obtenção do anel A (Esquema 27).

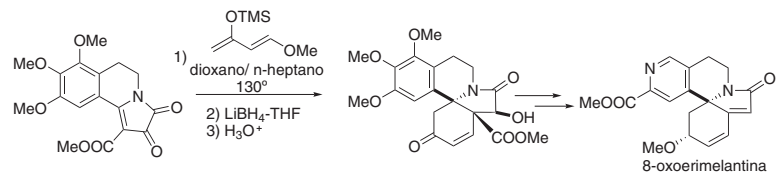

Esquema 27

A síntese da estrona tem como etapa chave a reação de DielsAlder entre o dieno de Dane e a metilciclopentenodiona, promovida pela catálise por um composto amidínio (Esquema 28). ${ }^{74}$

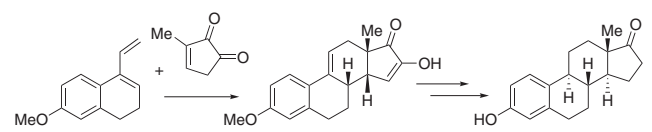

Esquema 28

A reação de Diels-Alder catalítica e assimétrica entre o dieno e o dienófilo, Esquema 29, foi utilizada na síntese de um intermediário na síntese do hiperforina. ${ }^{75}$

Uma reação de Diels-Alder intermolecular foi utilizada na síntese dos produtos naturais spirotenuipesinas A e B, por Danishefsky e colaboradores, ${ }^{76}$ levando à formação de um intermediário espiro (Esquema 30). 


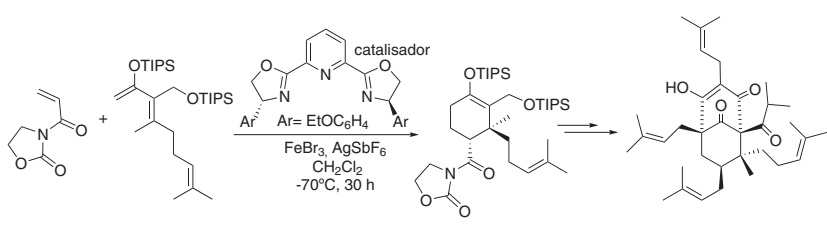

Esquema 29

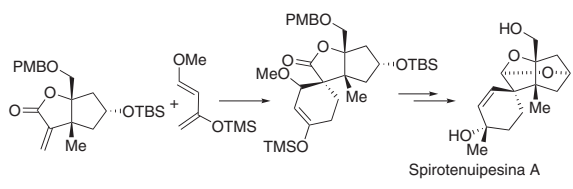

Esquema 30

\section{Reações intramoleculares (IMDA)}

Tadano e colaboradores, ${ }^{77}$ e depois Juhl e Tanner ${ }^{78}$ apresentaram excelentes revisões sobre a reação de Diels-Alder intramolecular (IMDA) e algumas recentes aplicações na síntese total de produtos naturais.

Pradilla e colaboradores apresentaram um exemplo de uma IMDA de dieninos sulfinilados quirais sob condições brandas, e com alta diastereosseletividade, na síntese de compostos hetero-bicíclicos e tricíclicos (Esquema 31). ${ }^{79}$

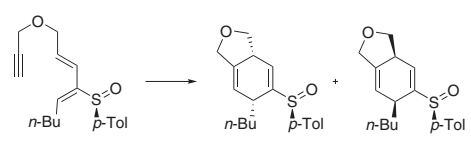

Esquema 31

Yadav e colaboradores descrevem a síntese de um intermediário chave para várias pseudopterosinas, utilizando uma reação de DielsAlder intramolecular de um dieno funcionalizado e um acetileno ligados por um espaçador com quatro átomos, formando um sistema tricíclico (Esquema 32). ${ }^{80}$

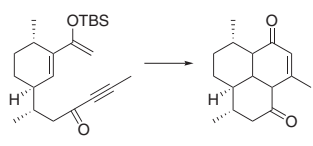

\section{Esquema 32}

O grupo de Dias relatou uma reação IMDA com a formação de produtos hidrindenos (Esquema 33), propícios para a síntese total da stawamicina. ${ }^{81}$

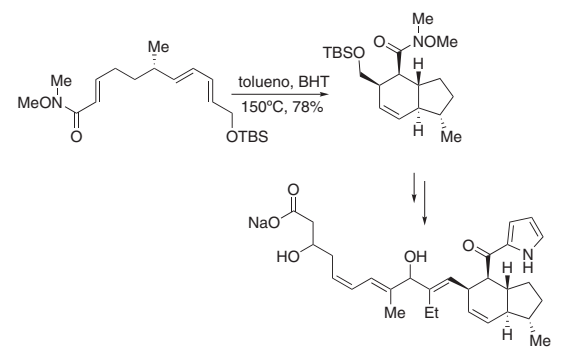

Esquema 33

\section{Reações transanulares (TADA)}

A reação de Diels-Alder transanular (TADA) é uma versão mais sofisticada da reação de Diels-Alder intramolecular (IMDA), utilizando uma estrutura macromolecular como substrato. Quando a análise conformacional deste sugere a aproximação das unidades do dieno e dienófilo, a reação ocorre conferindo ao cicloaduto pelo menos três anéis. ${ }^{1} \mathrm{O}$ desenvolvimento desta variação se deve basicamente ao grupo de Deslongchamps. ${ }^{82}$

O grupo de Jacobsen relata a reação TADA enantiosseletiva com elevado excesso enantiomérico, como etapa fundamental para a síntese de alguns sesquiterpenos (Esquema 34). ${ }^{83}$

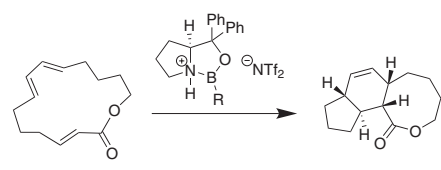

Esquema 34

Bodwell e colaboradores publicaram a síntese de um sistema pentacíclico contendo dois átomos de nitrogênio, obtido através de reação TADA de demanda inversa eletrônica (Esquema 35). ${ }^{84,85}$

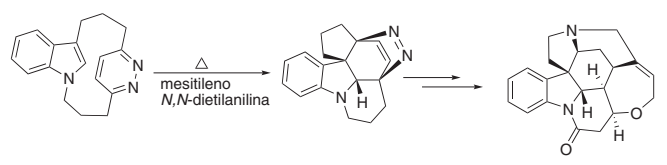

Esquema 35

O grupo de Deslongchamps relatou a síntese de compostos fluoroesteroides, uma nova classe não natural de cardenolidas, com elevada diastereosseletividade na reação TADA (Esquema 36) ${ }^{86}$

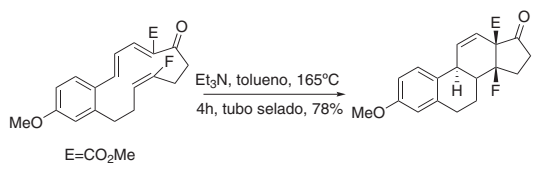

Esquema 36

Também o grupo de Deslongchamps realizou a síntese total da (+) Cassaina, utilizando esta estratégia para a formação do intermediário tricíclico (Esquema 37). ${ }^{87}$

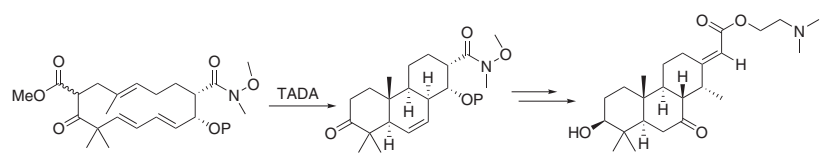

Esquema 37

Uma sequência dominó de duas reações de Diels-Alder transanular foi utilizada na síntese de FR182877, pelos grupos de Sorensen e Evans (Esquema 38). ${ }^{88-90}$

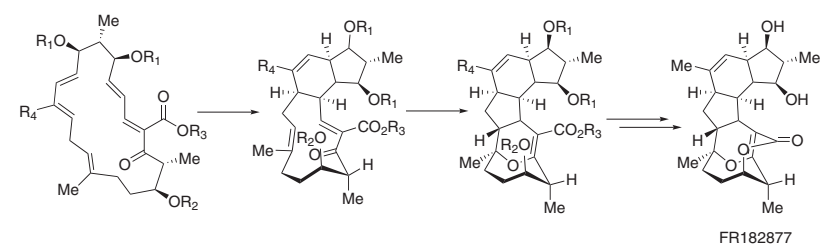

Esquema 38

Nakada e colaboradores publicaram em 2009, a síntese de (+)-fomopsidina através de uma reação TADA altamente estereosseletiva (Esquema 39). ${ }^{91}$ 


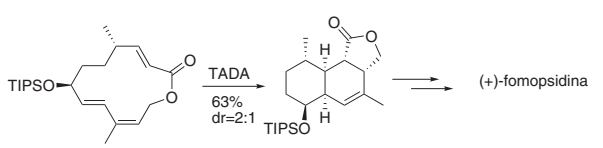

Esquema 39

\section{CONCLUSÃO E PERSPECTIVAS}

A reação de Diels-Alder continua sendo muito relevante em estudos mecanísticos, devido à sua complexidade no estado de transição. A questão de reações pericíclicas em biossíntese de produtos naturais está sendo confirmada a favor da participação com atividade enzimática. Novas metodologias estão surgindo constantemente e as suas utilizações em síntese total avançam logo a seguir. Certamente, esta situação deve continuar nas próximas décadas. As questões de quimiosseletividade, regiosseletividade e estereosseletividade, no sentido da inversão da tendência natural, estão sendo estudadas através do conceito de umpolung, e parece que podemos prever soluções importantes em breve. ${ }^{92}$

\section{AGRADECIMENTOS}

À FAPESP, CAPES e CNPq pelo apoio financeiro em auxílios e bolsas. Também agradecemos a colaboração fundamental de todos os alunos do laboratório em nossos estudos sobre a reação de DielsAlder.

\section{REFERÊNCIAS E NOTAS}

1. Brocksom, T. J.; Nakamura, J.; Ferreira, M. L.; Brocksom, U.; J. Braz. Chem. Soc. 2001, 12, 597.

2. Sci-Finder, página pesquisada em maio de 2010; a nossa escolha de publicações é realmente pessoal e reflete a nossa visão da relevância da reação e, como consequência, muitas novidades são omitidas.

3. Corey, E. J.; Angew. Chem., Int. Ed. 2002, 41, 1650.

4. Nicolaou, K. C.; Snyder, S. A.; Montagnon, T.; Vassilikogiannakis, G.; Angew. Chem., Int. Ed. 2002, 41, 1668.

5. Fringuelli, F.; Taticchi, A.; The Diels-Alder Reaction; Selected Practical Methods, Wiley: Chichester, 2001.

6. Anh, N. T.; Frontier Orbitals; a Practical Manual, Wiley: Chichester, 2007.

7. Fleming, I.; Molecular Orbitals and Organic Chemical Reactions, Wiley: Chichester, 2010.

8. Vasil'ev, A. A.; Serebryakov, E. P.; Russ. Chem. Rev. 2001, 70, 735.

9. Young, I. S.; Baran, P.; Nature Chemistry 2009, 216, 193.

10. Trost, B. M.; Science 1991, 254, 1471.

11. Wender, P. A.; Handy, S. T.; Wright, D. L.; Chem. Ind. 1997, 765; Wender, P. A.; Chem. Rev. 1996, 96, 1.

12. Hendrickson, J. B.; J. Am. Chem. Soc. 1975, 97, 5784; o conceito simplificado relaciona o número de reações construtivas $(\mathrm{nC}$, onde há a formação de ligações carbono-carbono) e o número total de reações (nT) numa sequência sintética; citado nas aulas de D. A. Evans, Harvard, Chem 30, Synthesis Strategy-2, p. 66, 1993.

13. Frederico, D.; Brocksom, U.; Brocksom, T. J.; Quim. Nova 2005, 28, 692.

14. Nicolaou, K. C.; Bulger, P. G.; Sarlah, D.; Angew. Chem., Int. Ed 2005, $44,4490$.

15. Nicolaou, K. C.; Bulger, P. G.; Sarlah, D.; Angew. Chem., Int. Ed. 2005, $44,4442$.

16. Nicolaou, K. C.; Montagnon, T.; Snyder, S. A.; Chem. Commun. 2003 551.

17. Nicolaou, K. C.; Edmonds, D. J.; Bulger, P. G.; Angew. Chem., Int. Ed. 2006, 45, 7134 .
18. Ver por exemplo: Constantino, M. G.; Química Orgânica; Curso Básico Universitário, LTC Editora: Rio de Janeiro, 2008, vol. 2, caps. 3.7 e 3.8; McMurry, J.; Organic Chemistry, Thomson: Belmont, 2008, caps. 14.4 e 30; Clayden, J.; Greeves, N.; Warren, S.; Wothers, P.; Organic Chemistry, Oxford University Press: Oxford, 2001, caps. 35 e 36.

19. Ver por exemplo: Carey, F. A.; Sundberg, R. J.; Advanced Organic Chemistry, 5a ed., Springer: New York, 2007; Smith, M. B.; March, J.; March's Advanced Organic Chemistry, 6ª ed., Wiley: Hoboken, NJ, 2007.

20. Lacerda, Jr. V.; de Oliveira, K. T.; Costa e Silva, R.; Constantino, M. G.; da Silva, G. V. J.; Quim. Nova 2007, 30, 727.

21. Reportagem em Chem. Eng. News, $29 / 11 / 2004,82$, n. 48, p. 5.

22. Hoffmann, R.; Angew. Chem., Int. Ed. 2004, 43, 6586.

23. Por exemplo: Domingo, L. R.; Aurell, M. J.; Pérez, P.; Contreras, R.; J. Org. Chem. 2003, 68, 3884.

24. Hoffmann, R.; Woodward, R. B.; Acc. Chem. Res. 1968, 1, 17 e refs. citadas.

25. Fukui, K.; Acc. Chem. Res. 1971, 4, 57.

26. García, J. I.; Mayoral, J. A.; Salvatella, L.; Acc. Chem. Res. 2000, 33, 658.

27. Arrieta, A.; Cossío, F. P.; Lecea, B.; J. Org. Chem. 2001, 66, 6178.

28. Yates, P.; Switlak, K.; Can. J. Chem. 1990, 68, 1894.

29. Tormena, C. F.; Lacerda Jr, V.; de Oliveira, K. T.; J. Braz. Chem. Soc. 2010, 21, 112.

30. Spino, C.; Rezaei, H.; Dory, Y. L.; J. Org. Chem. 2004, 69, 757.

31. Ogawa, A.; Fujimoto, H.; Tetrahedron Lett. 2002, 43, 2055.

32. Lam, Y-H.; Cheong, P. H-Y.; Blasco Mata, J. M.; Stanway, S. J.; Gouverneur, V.; Houk, K. N.; J. Am. Chem. Soc. 2009, 131, 1947.

33. Paddon-Row, M. N.; Christopher, D.; Anderson, C. D.; Houk, K. N.; J. Org. Chem. 2009, 74, 861.

34. Hayden, A. E.; DeChancie, J.; George, A. H.; Dai, M.; Yu, M.; Danishefsky, S. J.; Houk, K. N.; J. Org. Chem. 2009, 74, 6770.

35. Stocking, E. M.; Williams, R. M.; Angew. Chem., Int. Ed. 2003, 42, 3078 .

36. Oikawa, H.; Tokiwano, T.; Nat. Prod. Rep. 2004, 21, 321.

37. Kelly, W. L.; Org. Biomol. Chem. 2008, 6, 4483.

38. Watanabe, K.; Biosci. Biotechnol. Biochem. 2008, 72, 2491.

39. Gouverneur, V.; Reiter, M. ; Chem. Eur. J. 2005, 11, 5806.

40. Pohnert, G.; ChemBioChem. 2003, 4, 713.

41. Guimarães, W. C. R.; Udier-Blagovic, M.; Jorgensen, W. L.; J. Am. Chem. Soc. 2005, 127, 3577.

42. Nunes, R. L.; Bieber, L.W.; Tetrahedron Lett. 2001, 42, 219.

43. Constantino, M. G.; Júnior, V. L.; da Silva, G. V, J.; Molecules 2002, 7, 456.

44. Da Silva Filho, L. C.; Lacerda Jr, L. C. V.; Constantino, M. G.; da Silva, G. V. J.; Invernize, P. R.; Beilstein J. Org. Chem. 2005, 1, 14.

45. Huang, Y.; Unni, A. K.; Thadani, A. N.; Rawal, V. H.; Nature 2003, 424, 146.

46. Lelais, G.; MacMillan, D. W. C.; Aldrichimica Acta 2006, 39, 79.

47. Merino, P.; Marqués-López, E.; Tejero, T.; Herrera, R. P.; Synthesis 2010, 1.

48. Kristensen, T. E.; Vestli, K.; Jakobsen, M. G.; Hansen, F. K.; Hansen, T.; J. Org. Chem. 2010, 75, 1620.

49. Mitsudome, T.; Nose, K.; Mizugaki, T.; Jitsukawa, K.; Kaneda, K.; Tetrahedron Lett. 2008, 49, 5464.

50. Herrero, M. A.; Kremsner, J. M.; Kappe, C. O.; J. Org. Chem. 2008, 73, 36.

51. Obermayer, D.; Gutmann, B.; Kappe, C. O.; Angew. Chem., Int. Ed. 2009, 48, 8321.

52. Bowman, M. D.; Schmink, J. R.; McGowan, C. M.; Kormos, C. M.; Leadbeater, N. E.; Org. Process Res. Dev. 2008, 12, 1078.

53. Damm, M.; Glasnov, T. N.; Kappe, C. O.; Org. Process Res. Dev. 2010, $14,215$. 
54. Choshi, T.; Kumemura, T.; Nobuhiro, J.; Hibino, S.; Tetrahedron Lett. 2008, 49, 3725.

55. Songis, O.; Géant, J. Y.; Sautrey, G.; Martinez, J.; Calmès, M.; Eur. J. Org. Chem. 2008, 308, 308

56. Gómez, M. V.; Aranda, A. L.; Moreno, A.; Cossío, F. P.; Cózar, A.; DíazOrtiz, A.; de la Hoz, A.; Prieto, P.; Tetrahedron 2009, 65, 5328.

57. Castagnolo, D.; Botta, L.; Botta, M.; Tetrahedron Lett. 2009, 50, 1526.

58. Donatoni, M. C.; Brocksom, T. J.; 13th BMOS, São Pedro, 2009, p. 72.

59. Ramón, D. J.; Yus, M.; Angew. Chem., Int. Ed. 2005, 44, 1602.

60. Vieira, Y. W.; Nakamura, J.; Finelli, F. G.; Brocksom, U.; Brocksom, T. J.; J. Braz. Chem. Soc. 2007, 18, 448.

61. Tietze, L. F.; Chem. Rev. 1996, 96, 115.

62. Poulin, J.; Grisé-Bard, C. M.; Barriault, L.; Chem. Soc. Rev. 2009, 38, 3092 .

63. Nicolaou, K. C.; Chen, J. S.; Chem. Soc. Rev. 2009, 38, 2993.

64. Desimoni, G.; Tacconi, G.; Barco, A.; Pollini, G. P.; Natural Product Synthesis Through Pericyclic Reactions, American Chemical Society: Washington, DC, 1983.

65. Constantino, M. G.; de Oliveira, K, T.; Polo, E. C.; Silva, G. V. J.; Brocksom, T. J.; J. Org. Chem. 2006, 71, 9880.

66. Da Silva, F. de C.; Ferreira, S. B.; Kaiser, C. R.; Pinto, A. C.; Ferreira, V. F.; J. Braz. Chem. Soc. 2009, 20, 1478.

67. Brocksom, T. J.; Corrêa, A. G.; Naves, R. M.; Silva Jr., F.; Catani, V.; Ceschi, M. A.; Zukerman-Schpector, J.; Toloi, A. P.; Ferreira, M. L.; Brockson, U. Em Organic Synthesis: Theory and Applications; Hudlicky, T., ed.; JAI/Elsevier Science: Oxford, 2001, vol. 5, p. 39.

68. Dias, L. C.; Fernandes, A. M. A. P.; Zukerman-Schpector, J.; Synlett 2002, 100

69. Hsu, D.; Hsu, Po.; Lee, Y.; Liao, C.; J. Org. Chem. 2008, 73, 2554.

70. Min, S.; Danishefsky, S. J.; Angew. Chem., Int. Ed. 2007, 46, 2199.

71. Nishikawa, K.; Nakahara, H.; Shirokura, Y.; Nogata,Y.; Yoshimura, E.; Umezawa, T.; Okino, T.; Matsuda, F.; Org. Lett. 2010, 12, 904.

72. Li, Z.; Gao, Y.; Tang, Y.; Dai, M.; Wang, G.; Wang, Z.; Yang, Z. ; Org. Lett. 2008, 10, 3017.

73. Yoshida,Y.; Mohri, K.; Isobe, K.; Itoh, T.; Yamamoto, K.; J. Org. Chem. 2009, 74, 6010 .

74. Weimar, M.; Dürner, G.; Bats, J. W.; Göbel, M. W.; J. Org. Chem. 2010, 75, 2718.

75. Shimizu, Y.; Shi, S.; Usuda, H.; Kanai, M.; Shibasaki, M.; Angew. Chem., Int. Ed. 2010, 49, 1103.

76. Dai, M.; Krauss, I. J.; Danishefsky, S. J.; J. Org. Chem. 2008, 73, 9576.

77. Takao, K.; Munakata, R.; Tadano, K.; Chem. Rev. 2005, 105, 4779.

78. Juhl, M.; Tanner, D.; Chem. Soc. Rev. 2009, 38, 2983.

79. Pradilla, R. F.; Tortosa, M.; Castellanos, E.; Viso, A.; Baile, R.; J. Org. Chem. 2010, 75, 1517.

80. Yadav, J. S.; Bhasker, E. V.; Srihari, P.; Tetrahedron 2010, 66, 1997.

81. Dias, L. C.; Jardim, L. S. A.; Ferreira, A. A.; Soarez, H. U.; J. Braz. Chem. Soc. 2001, 12, 463.

82. Marsault, E.; Toro, A.; Nowak, P.; Deslongchamps, P.; Tetrahedron 2001, 57, 4243 .
83. Balskus, E. E.; Jacobsen, E. N.; Science 2007, 317, 1736.

84. Bodwell, G. J.; Li, J.; Angew. Chem., Int. Ed. 2002, 41, 3261.

85. Bodwell, G. J.; Li, J.; Org. Lett. 2002, 4, 127.

86. Beaubien, S.; Deslongchamps, P.; Can. J. Chem. 2006, 84, 29

87. Phoenix, S.; Reddy, M, S.; Deslongchamps, P.; J. Am. Chem. Soc. 2008, 130, 13989.

88. Evans, D. A.; Starr, J. T.; J. Am. Chem. Soc. 2003, 125, 13531.

89. Vosburg, D. A.; Vanderwal, C. D.; Sorensen, E. J.; J. Am. Chem. Soc. 2002, 124, 4552.

90. Vanderwal, C. D.; Vosburg, D. A.; Weiler, S.; Sorensen, E. J.; J. Am. Chem. Soc. 2003, 125, 5393.

91. Hayashi, N.; Suzuki, T.; Usui, K.; Nakada, M.; Tetrahedron 2009, 65, 888.

92. Hoffmann, R. W., Elements of Synthesis Planning, Springer-Verlag: Berlin, 2009, cap. 6 .

\section{REFERÊNCIAS SUPLEMENTARES}

Algumas revisões relevantes foram publicadas recentemente, e incluem informações e discussões importantes sobre a reação de Diels-Alder. Estas revisões não foram incluídas no texto porque o seu conteúdo não foi apresentado, mas são interessantes para o leitor poder completar os seus conhecimentos.

O mecanismo e aspectos teóricos - Ess, D. H.; Jones, G. O.; Houk, K. N.; Adv. Synth. Catal. 2006, 348, 2337.

\section{Metodologias}

Catálise com ácidos de Lewis - Andrade, C. K. Z.; Curr. Org. Synth. 2004, 1, 333; Fringuelli, F.; Piermatti, O.; Pizzo, F.; Vaccaro, L.; Eur. J. Org. Chem. 2001, 439; Loh, T-P.; Chua, G-L.; Chem. Commun. 2006, 2739; Taguchi, T.; Saito, A.; Yanai, H.; The Chemical Record 2007, 7, 167; Yamamoto, Y.; J. Org. Chem. 2007, 72, 7817; Shen, J.; Tan, C-H.; Org. Biomol. Chem., 2007, 6, 3229; Reymond, S.; Cossy, J.; Chem. Rev. 2008, 108, 5359.

Organocatálise enantiosseletiva - Amarante, G. W.; Coelho, F.; Quim. Nova 2009, 32, 469; Guillena, G.; Ramón, D. J.; Yus, M.; Tetrahedron: Asymmetry 2007, 18, 693; Bruckmann, A.; Krebs, A.; Bolm, C.; Green Chem. 2008, 10, 1131; Núñez, M. G.; García, P.; Moro, R. F.; Díez, D.; Tetrahedron 2010, 66, 2089; Enders, D.; Grondal, C.; Huttl, M. R. M.; Angew. Chem., Int. Ed. 2007, 46, 1570; Gruttadauria, M.; Giacalone, F.; Notoa, R.; Adv. Synth. Catal. 2009, 351, 33.

Micro-ondas - Kappe, C. O.; Angew. Chem., Int. Ed. 2004, 43, 6250; Kappe, C. O.; Chem. Soc. Rev. 2008, 37, 1127.

Reações multicomponentes (MCR) - Ver itens anteriores

Reações de Diels-Alder em dominó - Ver itens anteriores.

Aplicações em síntese total - Mulzer, J.; Castagnolo, D.; Felzmann, W.; Marchart, S.; Pilger, C.; Enev, V. S.; Chem. Eur. J. 2006, 12, 5992; Pellissier, H.; Tetrahedron 2009, 65, 2839; Bartók, M.; Chem. Rev. 2010, 110, 1663; Jørgensen, K. A.; Eur. J. Org. Chem. 2004, 2093; Kurti, L.; Czakó, B.; Strategic Applications of Named Reactions in Organic Synthesis, Elsevier Academic Press: San Diego, 2005, p. 140-141. 